\title{
Inclusion Exclusion Criterion Data Collection Day
}

National Cancer Institute

\section{Source}

National Cancer Institute. Inclusion Exclusion Criterion Data Collection Day. NCI

Thesaurus. Code C83296.

The day of the week inclusion exclusion criterion data was collected. 